\title{
A new automatic method for estimating the peak auroral emission height from all-sky camera images
}

\author{
D. K. Whiter ${ }^{1}$, B. Gustavsson ${ }^{2, *}$, N. Partamies ${ }^{1}$, and L. Sangalli ${ }^{3}$ \\ ${ }^{1}$ Finnish Meteorological Institute, Helsinki, Finland \\ ${ }^{2}$ School of Physics and Astronomy, University of Southampton, Southampton, UK \\ ${ }^{3}$ Royal Military College of Canada, Kingston, Ontario, Canada \\ *now at: EISCAT Scientific Association, Kiruna, Sweden
}

Correspondence to: D. K. Whiter (daniel.whiter@fmi.fi)

Received: 4 September 2012 - Published in Geosci. Instrum. Method. Data Syst. Discuss.: 25 October 2012

Revised: 14 February 2013 - Accepted: 15 February 2013 - Published: 7 March 2013

\begin{abstract}
This paper presents a new fully automatic method for quickly finding the average peak emission height of a single auroral structure from a pair of all-sky camera images with overlapping fields of view. The peak emission height of the aurora must be estimated in order to calculate several other important parameters, such as horizontal spatial scales, optical flow velocities, and ionospheric electric fields. In most cases the height is not measured, but a value is assumed, often about $110 \mathrm{~km}$. It is unclear how accurate this assumption is. A future statistical study of the auroral height in which the method presented here will be applied to many years of observations will lead to more accurate assumptions of the height with quantitative error estimates, and therefore more accurate estimates of parameters derived using these assumed auroral heights. In the present work the performance of the new method is compared to another recent automatic method. It is found that the new method measures the peak emission height regardless of the shape of the volume emission rate profile, unlike the other recent method. However, the new method is less suitable than the other method for analysis of very wide auroral arcs (>30 km) or for aurora in the magnetic zenith of one of the images.
\end{abstract}

\section{Introduction}

An estimate of the height of maximum auroral emission is often needed for the derivation of other parameters, such as the widths of auroral structures, optical flow velocities, ionospheric electric field estimates, and electron precipitation en- ergies (e.g. Kaila, 1989; Knudsen et al., 2001; Whiter et al., 2008; Dahlgren et al., 2009; Partamies et al., 2010; Obuchi et al., 2011). However, the height of the aurora is rarely measured; often when a numerical value is required it is assumed, typically at about $110-130 \mathrm{~km}$ for the most prominant emission at $557.7 \mathrm{~nm}$. The accuracy of these assumptions is generally not well known.

This paper presents a new automated method for determining the peak emission height of the aurora from a pair of simultaneous all-sky camera images with partially overlapping fields of view. The motivation for this work is to develop a method suitable for performing a large statistical study of the height of the aurora in which hundreds of thousands of images will be analysed. Such a statistical study is expected to substantially improve estimates of the height under different conditions and the uncertainty introduced by assuming a value for the height in the derivation of other parameters. The peak emission height of the aurora depends on the energy of electron precipitation producing the aurora and the concentration profiles of the major neutral species in the thermosphere (e.g. Rees, 1963b; Judge, 1972). Long-term change and periodicity in these parameters could be identified using such a large statistical study of the height of the aurora.

In this work the performance of the new method and another recent automatic method based on 2-D-correlation (Ashrafi et al., 2005) are evaluated and compared using a set of synthetic images. To allow completion of the statistical study in a reasonable amount of time, both methods are fast, requiring about $5-10 \mathrm{~s}$ to analyse a pair of images on a typical modern computer (as of 2012), and both methods are 
automatic (for single arcs) and require no manual input during the analysis. It is desirable that the method used in the statistical study can successfully analyse all possible shapes and types of aurora. The present work examines the accuracy of the methods when analysing a variety of shapes and sizes of a single auroral structure located between two stations.

\section{Instrumentation}

The MIRACLE (Magnetometers - Ionospheric Radars All-sky Cameras Large Experiment) network includes several (between 5 and 8, depending on year) all-sky cameras distributed across northern Fenno-Scandinavia and Svalbard (Syrjäsuo et al., 1998) with overlapping fields of view. The all-sky cameras have been in continuous winter operation since 1996, and have produced many thousands of images of the aurora $\left(\sim 10^{5}\right.$ images per station per season), making them particularly suited for a statistical study into the height of the aurora. In this work three of the cameras, stationed at Abisko $\left(68.36^{\circ} \mathrm{N}, 18.82^{\circ} \mathrm{E}\right)$, Kilpisjärvi $\left(69.01^{\circ} \mathrm{N}, 20.79^{\circ} \mathrm{E}\right)$ and Sodankylä $\left(67.42^{\circ} \mathrm{N}, 26.39^{\circ} \mathrm{E}\right)$, have been used. Initially the MIRACLE cameras used intensified charge-coupled device (ICCD) detectors, but these were upgraded to a mixture of colour CCDs and electronmultiplying CCD (EMCCD) detectors in 2006 and 2007. In this work only ICCD data are used, but data from the EM$\mathrm{CCD}$ observations will also contribute to the future statistical study.

The ICCD and later EMCCD all-sky cameras are each equipped with a filter wheel and set of narrow passband $(\sim 2 \mathrm{~nm})$ interference filters for observing auroral emission at $630.0 \mathrm{~nm}$ ("red"), $557.7 \mathrm{~nm}$ ("green"), and $427.8 \mathrm{~nm}$ ("blue"), together with panchromatic images. The filter wheels attached to the EMCCD cameras also include filters for observing background emission. Only green images have been used in this work, but it is expected that the statistical study will involve all filters. The imaging sequence can be modified but is synchronised across all MIRACLE stations such that all cameras are observing in the same wavelength at the same time. Generally the cameras have produced a green image at least once per $20 \mathrm{~s}$ and blue and red images at least once per minute. The exposure time of the ICCD images has usually been $1 \mathrm{~s}$ for green and panchromatic images and $2 \mathrm{~s}$ for blue and red images.

\section{Previous work}

Sangalli et al. (2011) used a manual triangulation method to find the peak emission height of an auroral arc observed with the same all-sky cameras as are used in the present work. They found the peak emission height of the arc varied by about $40 \mathrm{~km}$ along its length, and the mean height varied between $120 \pm 10 \mathrm{~km}$ and $140 \pm 10 \mathrm{~km}$ over a period of $6 \mathrm{~min}$. Although the method they used gives information about the variation in height along auroral arcs, it is not ideal for a large-scale statistical study as it is time consuming and requires considerable manual input, prompting the development of the new method presented here. The new method does not give information about the variation in height along an arc, but is fast and automated.

Several workers developed methods to identify the height of the aurora during the 1960s. Naturally these methods were applied manually, although some could now be automated to take advantage of modern computers. However, modern computers allow the application of other methods (such as the new method presented here) which would have been prohibitively time consuming in the 1960s.

Roach et al. (1960) used a triangulation method to find the height of exceptional "monochromatic" red aurora at low latitude using three all-sky cameras. They found a peak emission height of $412 \pm 23 \mathrm{~km}$. The method relies on the assumption that the auroral arc lies along parallels of equal magnetic dip angle, which is valid for the exceptional red arcs but renders the method unsuitable for a statistical study in the Arctic. Rees (1963a) developed a method for determining the height of similar auroral forms to Roach et al. (1960) from a single all-sky camera image. The main disadvantage of this method is that it only works for arcs which are exactly east-west aligned. This assumption is most valid for arcs at relatively low latitude (for which this method was developed) with heights well above $100 \mathrm{~km}$, and is therefore not suitable for application to the MIRACLE data or for a statistical study.

A method for determining the height of aurora, which involves measuring the parallax between corresponding images from a pair of simultaneous wide-angle (nearly all-sky) cameras, was used by Brandy and Hill (1964). This method relies on accurately identifying the lower border of a welldefined arc, and gives the height of this lower border rather than the height of peak emission. Similar parallactic photography methods were used many years earlier with narrower field-of-view cameras (e.g. Störmer, 1955; Harang, 1951; McEwen and Montalbetti, 1958). Boyd et al. (1971) discussed the reasons for measuring the peak emission height rather than the height of the lower border of the arc. The peak emission height is more closely related to the energy of the precipitating electrons than the height of the lower border of the arc (Rees, 1963b). Also, the location of the lower border is dependent on the sensitivity of the observing instrument, and therefore combining measurements from different instruments can produce incorrect results for the height of the aurora, especially if the instruments are of different ages. The new method presented in this work is designed to measure the height of peak emission, and so does not suffer from these problems.

Romick and Belon (1967a) evaluated the accuracy of a triangulation method for finding the height of maximum auroral intensity from a pair of meridian scanning photometer (MSP) observations separated by $226 \mathrm{~km}$ and located on the same 

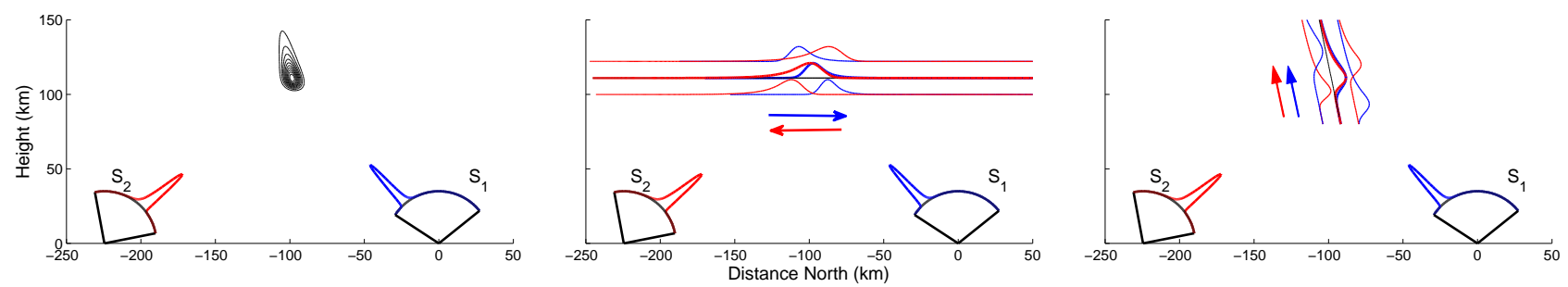

Fig. 1. Cartoon showing the projection of images of an auroral arc located between two stations (shown in left panel) onto three different horizontal planes (middle panel) and three different geomagnetic field lines (right panel). The colour-coded arrows in the middle and right panels indicate the "up" direction in each projection. This shows that in the case of the horizontal plane the shape of the two projections is flipped.

geomagnetic meridian. They produced a mathematical formulation for synthetic auroral arcs as viewed by MSPs, and tested the triangulation method for different arc positions, widths, and height profiles. They found that the method overestimated the height when the arc was located between the two stations, and underestimated the height for arcs which were far away from both stations, but worked well when the arc was overhead one of the two stations. They also suggested an iterative technique to determine volume emission rate profiles of quiet auroral arcs using this method, which was used in a second paper (Romick and Belon, 1967b).

Boyd et al. (1971) used the triangulation method tested by Romick and Belon (1967a) for identifying the peak auroral emission height from a system of three meridian scanning photometers located on the same dipole meridian. The scanning photometer instrumentation restricts their study to quiet and homogeneous structures. Other workers also used this technique, but applied to two photometers only (e.g. Sandholt et al., 1982; Sigernes et al., 1996; Deehr et al., 2005). Sigernes et al. (1996) emphasise that their analysis is restricted to narrow auroral structures. Jackel et al. (2003) argued that a good angular resolution is required for this technique, as the resulting height measurement is sensitive to errors in the angle of the intensity peak. Instead they developed a technique in which scans from a pair of meridian scanning photometers are projected onto an invariant latitude grid at a range of assumed heights. The height which produces the largest correlation coefficient between the two scans is taken as the peak emission height. Ashrafi et al. (2005) expanded the method to 2-dimensional camera images. A fast and automatic version of this method is evaluated in this work, and is described fully in Sect. 4.1 below.

Tomographic-like inversion is another technique which has recently been used for finding the height of atmospheric emission from camera images (e.g. Frey et al., 1996; Aso et al., 1998; Gustavsson, 2000; Tanaka et al., 2011). However, this method is not ideal for a statistical study as it requires too time-consuming computations and manual input to preprocess the images. Also, to be accurate it requires images of the same structure observed from many stations, which is not often possible due to varying local weather con- ditions and cloud cover at the different stations. This would therefore reduce the size of a statistical study.

With the exception of the method used by Ashrafi et al. (2005), none of the above methods are suitable for a statistical study using all-sky cameras either because they are not fully applicable to the instrumentation, they are too timeconsuming, or they are not sufficiently accurate when applied to a range of different auroral structures (including measuring the lower border of the arc rather than the peak emission height).

\section{Methods and analysis}

The two methods used in this work to determine the auroral peak emission height both involve projecting or "remapping" overlapping images from a pair of stations and maximising the correlation between the remapped images. The main difference between the two methods is the type of remapping. Method 1 is a version of the method used by Ashrafi et al. (2005) in which images are mapped onto a horizontal plane. The second method is novel and has not been previously used to estimate the height of atmospheric emission. In this new method images are mapped onto the Earth's magnetic field lines.

\subsection{Method 1: horizontal plane}

This method involves projecting simultaneous all-sky camera images from two stations onto a horizontal plane at a given height. A 2-dimensional linear Pearson correlation coefficient is calculated between the two images for the portions which are overlapping. This is repeated for different heights of the horizontal plane, and the height which leads to the largest correlation coefficient is taken to be the height of the aurora. In the version of the technique used here, heights from $80 \mathrm{~km}$ to $270 \mathrm{~km}$ are initially tested at $10 \mathrm{~km}$ separation before a second iteration tests heights with a separation of $1 \mathrm{~km}$ at up to $10 \mathrm{~km}$ either side (higher or lower) of the initial result. This iterative procedure reduces the computational time required in the analysis. 

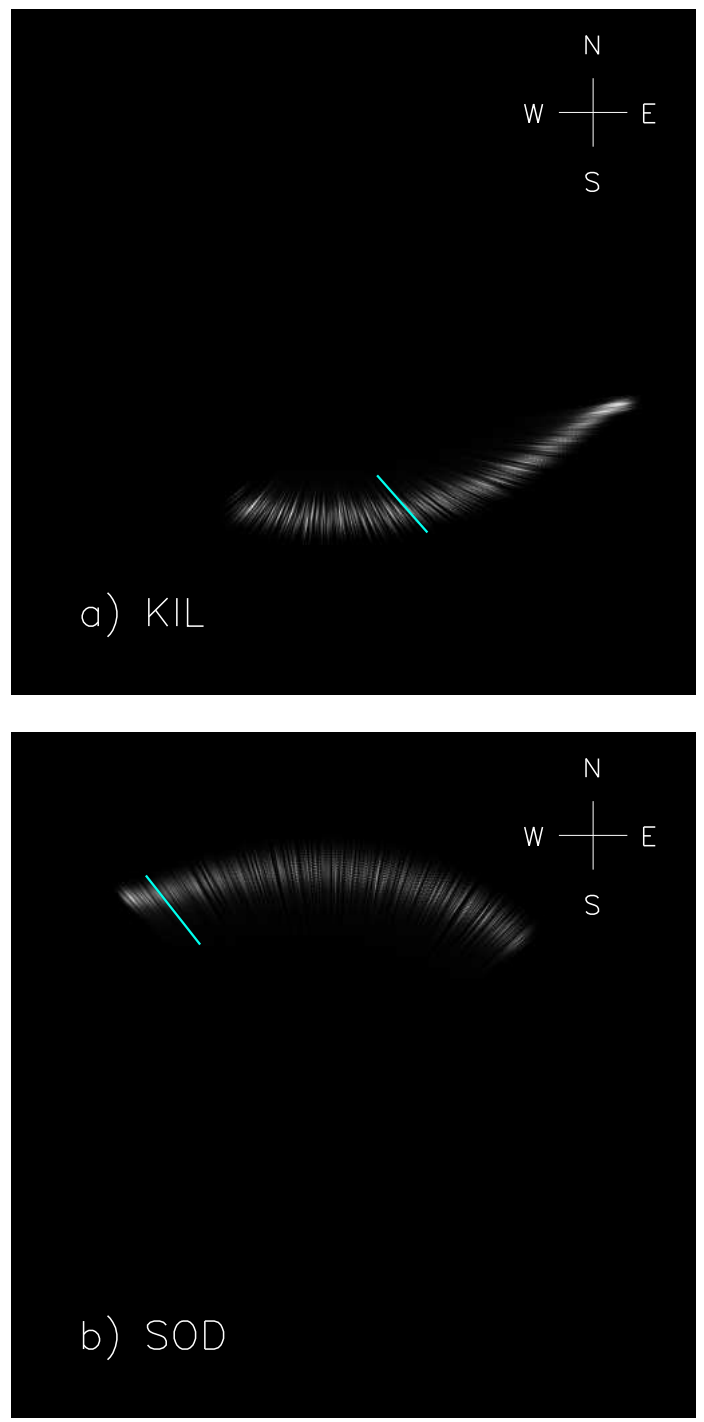

Fig. 2. Synthetic images of the same arc for all-sky cameras in (a) Kilpisjärvi and (b) Sodankylä. A single geomagnetic field line is highlighted in cyan.

The method is illustrated in the middle panel of the 2dimensional illustration shown in Fig. 1. Images of an auroral arc observed by two stations, $S_{1}$ (blue) and $S_{2}$ (red), are shown projected onto a horizontal plane at three different heights. On the central plane the images (red and blue brightness curves) are most highly correlated, and so the height of this plane is taken as the peak emission height of the aurora. Figure 1 is explained in more detail later in this paper.

\subsection{Method 2: magnetic field lines}

In this method the all-sky camera images are projected onto individual magnetic field-lines rather than a horizontal plane. The basic concept behind this is illustrated in Fig. 2, which shows synthetic images of a single thin auroral arc viewed simultaneously by the Kilpisjärvi (a) and Sodankylä (b) MIRACLE stations. The method for generating these synthetic images is described later in Sect. 5. Projecting a 2dimensional image onto a single field-line is equivalent to taking a curved cut across the image. In Fig. 2, one geomagnetic field line is marked in cyan in the altitude range 80$200 \mathrm{~km}$. One end of the cut corresponds to the bottom of the field line ( $80 \mathrm{~km}$ altitude), while the other end of the cut corresponds to the top ( $200 \mathrm{~km}$ altitude). The same field line is highlighted in the images from Kilpisjärvi and Sodankylä.

When an image of an arc is projected onto a field line whose geographic position (latitude and longitude) coincides with that of the arc, the field line cut across the image can be used to extract an emission height profile for the arc at that location. Figure 3a shows the emission altitude profile extracted from the Kilpisjärvi (red) and Sodankylä (blue) images for the field line marked in Fig. 2. The field line lays within the simulated arc, and the profiles from the two cameras correlate well (correlation coefficient 0.99). It is likely that the two profiles would not correlate well if the field line was outside the arc. Method 2 examines many field lines at different locations and uses three tests (described below) to identify which field lines lay within (or very close to) the auroral structure. Figure $3 \mathrm{~b}$ shows the emission altitude profile for the whole arc, created by averaging together the altitude profiles for all field lines which passed the three tests. The peak height is found to be $110 \mathrm{~km}$, which is equal to the true peak height of the synthetic arc.

The different outcomes for "good" field lines located very close to an auroral structure and those located further away are illustrated in the right panel of Fig. 1, simplified in 2 dimensions. The images of the arc from $S_{1}$ (blue) and $S_{2}$ (red) are shown projected onto three different field lines at different locations. On the central field line the two projections are very similar and highly correlated. Projecting the images onto the other two field lines shown in the illustration do not lead to profiles which are highly correlated, and therefore these profiles would not pass the three tests and would not be included in the mean volume emission rate profile for the auroral structure.

The details of the method are shown in Fig. 4, which demonstrates its application to the same synthetic auroral structure as shown in Fig. 2. A set of magnetic field lines is generated across the most significant bright feature within the overlapping portions of the images, which is identified using a connectivity-based clustering algorithm. The brightest $5 \%$ of pixels within one of the images are grouped into clusters such that the euclidean distance between neighbouring pixels in the same cluster is no more than twice the shortest distance between any two of the bright pixels. The cluster containing the largest number of bright pixels is selected to represent the most significant structure within the image. The outline of the selected region is shown by a white box in panels (a) Kilpisjärvi and (b) Sodankylä of Fig. 4. The positions of test field lines within this region are random, following a 

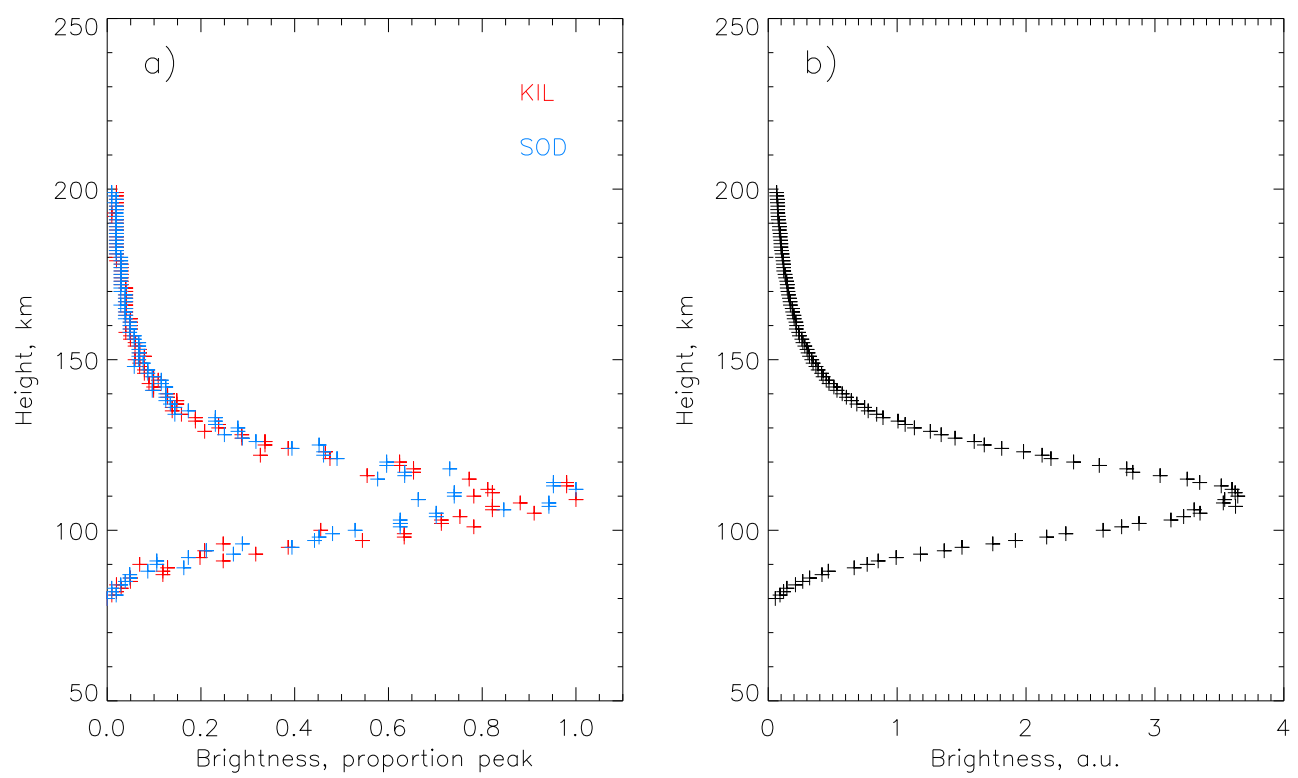

Fig. 3. (a) Altitude profile extracted from the field line highlighted in Fig. 2 for Kilpisjärvi (red) and Sodankylä (blue). (b) Average over all field lines which pass the three tests. The brightness is shown in arbitrary units (a.u.).

uniform distribution with a mean spacing of $0.05^{\circ}$ in latitude and $0.075^{\circ}$ in longitude at $100 \mathrm{~km}$ altitude $(\sim 5 \mathrm{~km}$ separation). The positions are random because it was found that if they conformed to a regular grid, the result could be biased to either lower or higher altitudes if the arc lay approximately parallel, but slightly away from, one row of the grid. A selection of 10 random field line footprints are marked with coloured crosses in the top panels of Fig. 4. The field lines at each point are traced between $80 \mathrm{~km}$ and $200 \mathrm{~km}$ altitude using the 10th generation International Geomagnetic Reference Field (IGRF-10) model (Maus et al., 2005). The images are projected onto each of these field lines individually, such that each field line corresponds to a curved cut across each image, as shown in the middle panels of Fig. 4. Those field lines which successfully pass three specific tests are averaged together to produce a mean emission altitude profile for the structure. The height of peak emission is taken from this mean altitude profile. The three tests are as follows:

1. The correlation coefficient between the projections from the two images must be greater than 0.5 .

2. The field line must show a clear peak in the brightness altitude profile (peak brightness substantially higher than the means of both the upper and lower portions of the field line. The required peak to mean ratio varies depending on the overall brightness of the structure, in order to work with different types of image).

3. The seperation between the luminosity height profile averages (analogous to centre of mass) of the two projections must be smaller than $20 \mathrm{~km}$.
After analysis under these conditions, a second iteration is performed in which the mean spacing between field lines is reduced by a factor 3 and only the regions surrounding field lines which "passed" the first iteration are considered. For this second iteration the tests are made more stringent; the correlation coefficient must be greater than 0.7 and the spread between the luminosity height profile averages must be smaller than $1 \mathrm{~km}$. The analysis is performed over 2 iterations in this way to reduce the computational time required. The bottom panels of Fig. 4 show the altitude profiles extracted from the dark blue (left) and dark red (right) field line cuts. The profiles from the Kilpisjärvi and Sodankylä images are shown as solid and dashed lines, respectively. The brightness scale is arbitrary. The horizontal black lines mark the luminosity height profile averages. In the case of the dark blue field line (left panel), the luminosity height profile averages of the Kilpisjärvi (solid) and Sodankylä (dashed) profiles are close, and the field line passes the tests for inclusion in the average profile. However, in the case of the dark red field line (right panel), the separation between the luminosity height profile averages is quite large, and so this field line is rejected in the second iteration.

\section{Synthetic images}

A set of 9310 pairs of synthetic images has been created to test the two analysis methods. Each pair of synthetic images shows a single auroral arc as viewed from the Sodankylä and Kilpisjärvi MIRACLE stations. In all cases the centre of the arc is located between the two stations. The performance of 

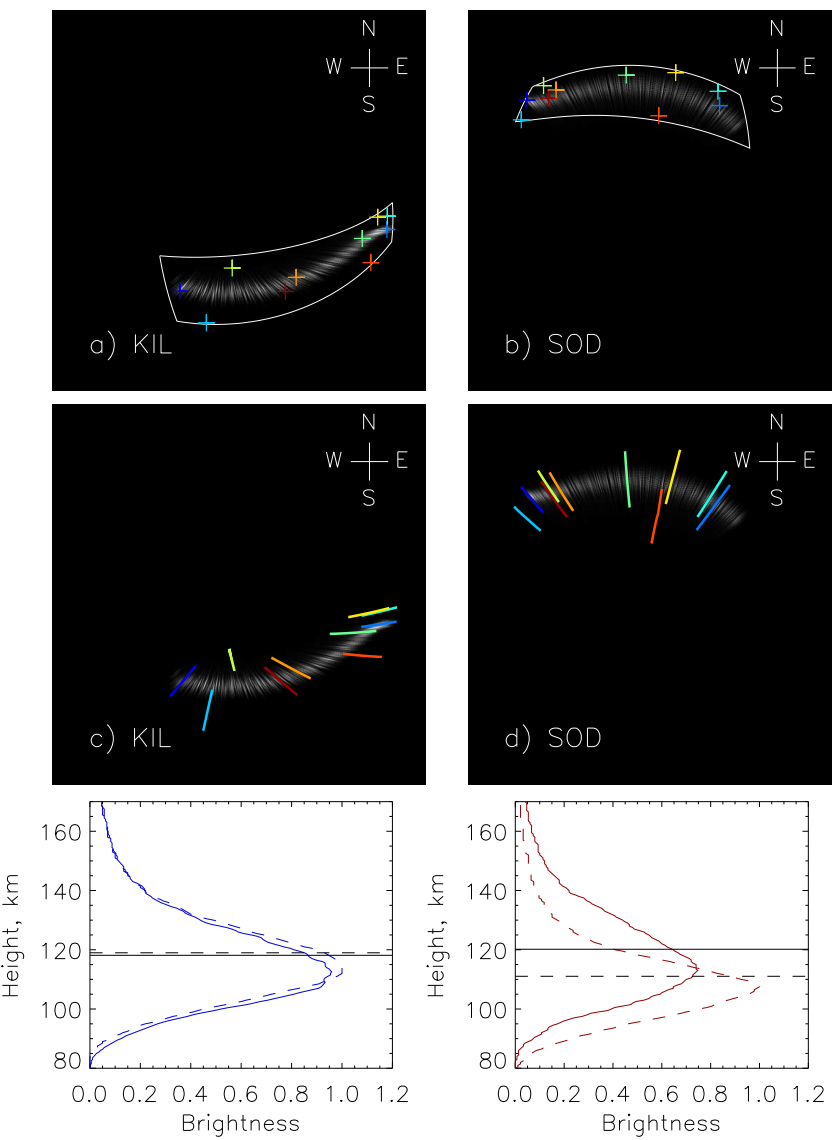

Fig. 4. The testing of different magnetic field lines as part of method 2. Ten selected field lines are shown on synthetic images from the Kilpisjärvi and Sodankylä stations. Top: the footprints of the test field lines at $100 \mathrm{~km}$ height. Middle: the projections of the field lines as cuts on the images. Bottom: height profiles for a "successful" test field line (dark blue, left), and a "failed" test field line (dark red, right).

each method can be evaluated as the emission height profile of the simulated arcs is known.

The process of generating a pair of synthetic images is illustrated in Fig. 5. Firstly, a rectangular grid of regularly spaced auroral ray "footprints" is generated, as shown in Fig. 5a. These footprints are defined with an arbitrary altitude of $100 \mathrm{~km}$, in a N-S/E-W cartesian coordinate system (hence the curvature of the grid when plotted in the latitudelongitude coordinate system of Fig. 5a). Each footprint is $2.5 \mathrm{~km}$ from each of its neighbours. The length $(450 \mathrm{~km})$ and width $(10 \mathrm{~km})$ of the grid of ray footprints defines the length and width of the resulting simulated arc. The locations of the Sodankylä and Kilpisjärvi observing stations are marked in Fig. 5 with blue and red points respectively. Curvature is added to the grid of footprints (Fig. 5b) to more accurately represent a real arc, according to a "bendiness" parameter (described below). Next, the grid of footprints is rotated (Fig. 5c) before each footprint is shifted a small ran-
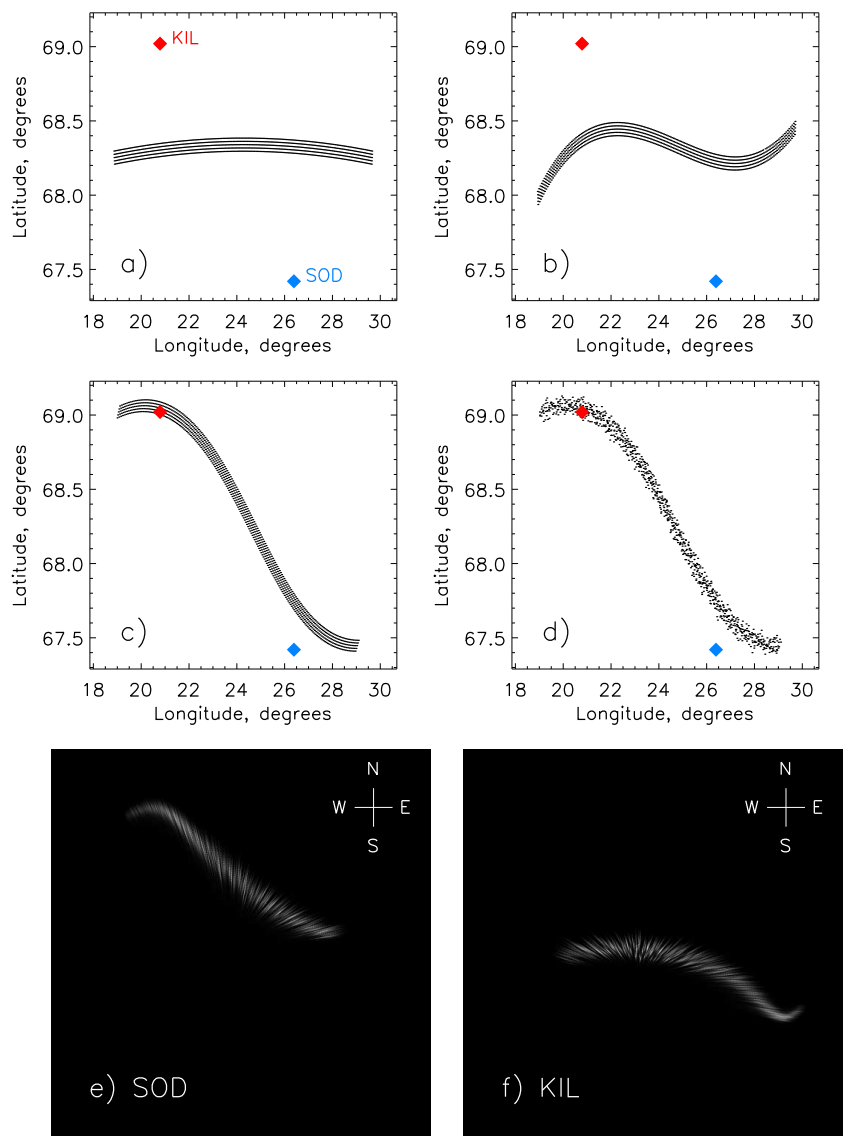

Fig. 5. The process for generating synthetic images. (a) Initial grid of ray footprints. The red and blue points mark the locations of Kilpisjärvi and Sodankylä respectively. (b) Grid of footprints after applying curvature. (c) After rotation. (d) After adding "noise". (e) The resulting arc as viewed from Sodankylä. (f) The resulting arc as viewed from Kilpisjärvi.

dom distance in a random direction to add noise to the grid (Fig. 5d). The final step is to apply the estimated emission altitude profile shown in Fig. 6 to each auroral ray footprint and to map the emission to the image plane of each camera. The magnetic field line passing through each footprint at $100 \mathrm{~km}$ is traced using the IGRF-10 model (Maus et al., 2005) to estimate the 3-dimensional shape of the auroral rays. The emission height profile shown in Fig. 6 was created by scaling an atomic oxygen concentration profile from the MSISE-90 model (Hedin, 1991) so that the peak concentration (emission) is at exactly $110 \mathrm{~km}$. Example synthetic images are shown in Fig. 5e and $\mathrm{f}$ for the Sodankylä and Kilpisjärvi stations, respectively, generated from the footprints shown in Fig. 5d.

The shape of each synthetic arc is defined by a set of four parameters: length, width, angle, and "bendiness". In the set of 9310 pairs of synthetic images used in this work, each pair has a different combination of arc parameters. The length ranges from $50 \mathrm{~km}$ to $700 \mathrm{~km}$ in steps of $50 \mathrm{~km}$. The 


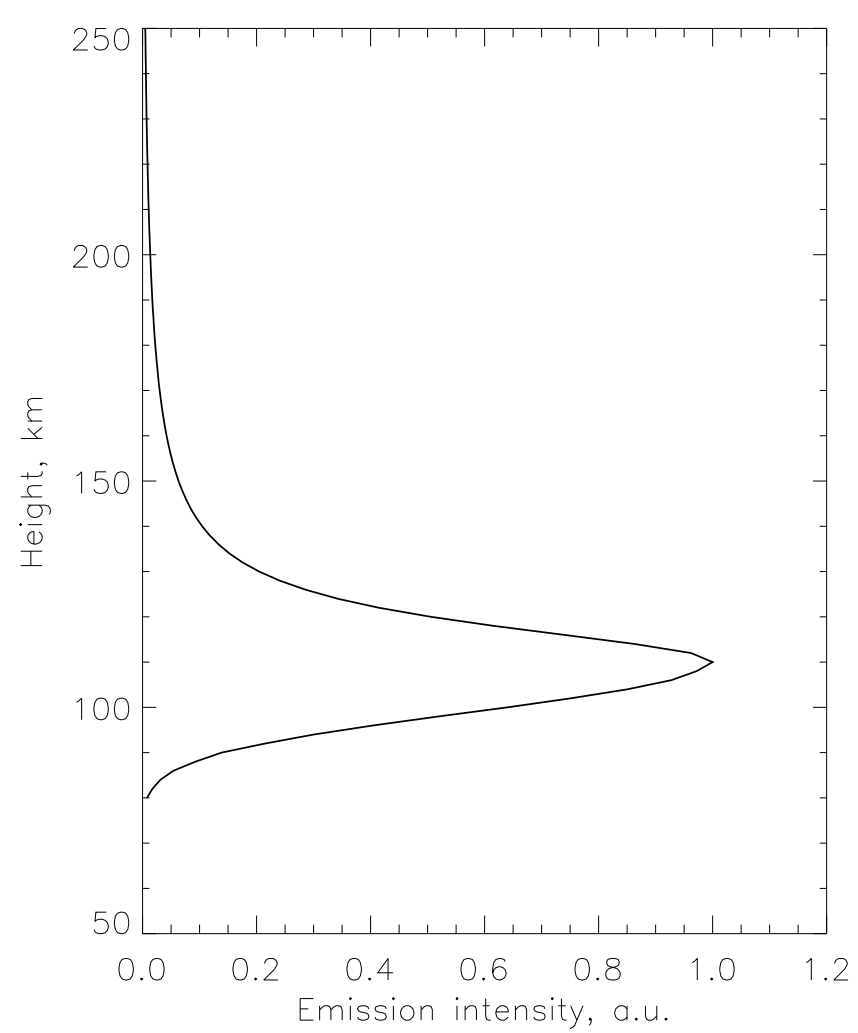

Fig. 6. Volume emission rate profile with a peak at $110 \mathrm{~km}$, used when generating the synthetic images.

width ranges from $5 \mathrm{~km}$ to $35 \mathrm{~km}$ in steps of $5 \mathrm{~km}$. The angle ranges from $0^{\circ}$ (east-west aligned) to $90^{\circ}$ (north-south aligned) in steps of $5^{\circ}$ (clockwise looking down). The bendiness ranges from 0.05 to 0.25 in steps of 0.05 . The curve of the arc is made up of a combination of sinusoidal and polynomial components designed to produce a variety of shapes while accurately representing an auroral arc. The sinusoidal and polynomial components are functions of the bendiness parameter as well as random values. Therefore the bendiness parameter cannot reproduce an exact arc shape, but controls how straight the arc is, with a value of zero corresponding to a perfectly straight arc. Examples of two arcs with different bendiness parameters are shown in Fig. 7. This figure approximately illustrates the extremes of curvature of the synthetic arcs used in this study, although it should be noted that as the shape of the arc has some dependence on random values, it is probable that both straighter and more curved arcs form part of the set. Figure $7 \mathrm{a}$ and $7 \mathrm{~b}$ show the location of the ray footprints for simulated arcs with bendiness 0.05 and 0.25 , respectively. Figure $7 \mathrm{c}$ and d show these two arcs as viewed from Sodankylä. In both cases the arcs are $450 \mathrm{~km}$ long, $10 \mathrm{~km}$ wide, and have an angle of $10^{\circ}$ with respect to a circle of latitude.

The range of auroral arc lengths within the set of synthetic images $(50-700 \mathrm{~km})$ provides an accurate representation of
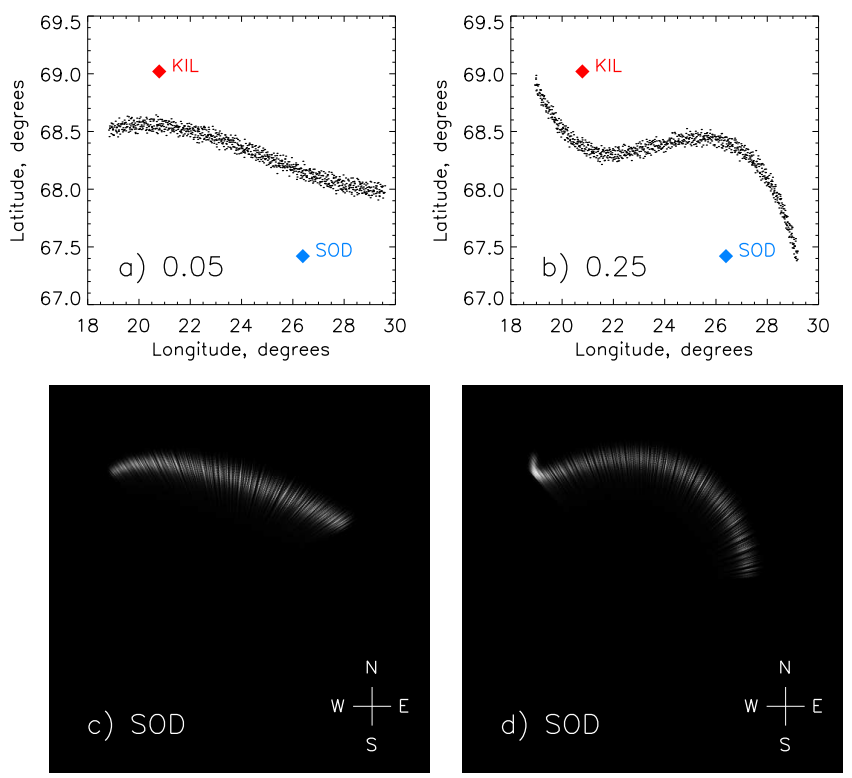

Fig. 7. (a) Ray footprints for a simulated arc with bendiness 0.05 . (b) Ray footprints for a simulated arc with bendiness 0.25. Panels (c) and (d) show the arcs in panels (a) and (b) respectively, as viewed from Sodankylä.

real observations; arcs of length $700 \mathrm{~km}$ reach close to the edge of the field-of-view at both ends of the arc and at both stations, representing large-scale structures which are only partially observed by the cameras, while the shorter lengths represent smaller, isolated structures. The range of arc widths $(5-35 \mathrm{~km})$ is representitive of a continuous distribution with a lower limit cutoff at $5 \mathrm{~km}$ due to the instrument resolution (Partamies et al., 2010). While Knudsen et al. (2001) found arc widths larger than $35 \mathrm{~km}$, this result is likely biased towards larger widths due to the aspect angle problem (Semeter et al., 2008; Partamies et al., 2010). Also, Knudsen et al. (2001) defined arc widths at $135 \mathrm{~km}$ altitude, and so these widths will be slightly larger than those here which are defined at $100 \mathrm{~km}$. The true distribution of arc angles will be dominated by east-west aligned arcs (angles close to $0^{\circ}$ ) with a small north-south aligned component (Syrjäsuo and Donovan, 2005). However, ideally the method for finding the height of an auroral structure would work well for all possible arc angles, and so the set of synthetic images is not dominated by any particular direction. Arc angles between $90^{\circ}$ and $180^{\circ}$ have not yet been tested, although it is expected that both methods would perform best on these arcs as they would lie aproximately perpendicular to the baseline between the two stations and so would have a minimum length-height ambiguity. 

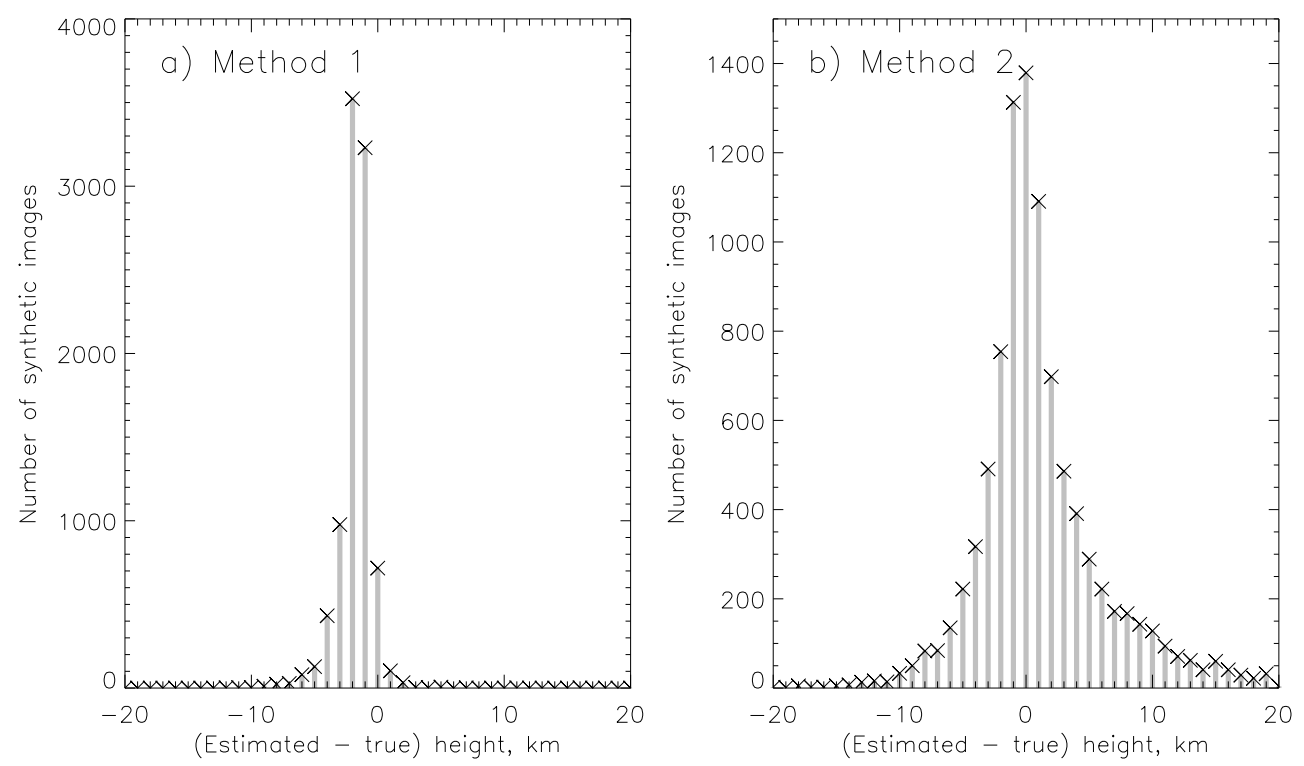

Fig. 8. Histograms showing the results from applying method 1 (a) and method 2 (b) to the set of synthetic images. Standard deviations: (a) 1.3 , (b) 4.8 .

\section{Results and discussion}

The two methods described in Sect. 4 have been evaluated using the set of synthetic images described in Sect. 5. The height produced by the two methods has been compared to the true peak height of the synthetic arcs, which is fixed at $110 \mathrm{~km}$. Figures $8 \mathrm{a}$ and $\mathrm{b}$ shows histograms of the difference between the found height and the true height for methods 1 and 2 respectively. The narrow distribution in Fig. 8a ( standard deviation $=1.3 \mathrm{~km}$ ) shows that the horizontal plane method gives a consistent result. However, the peak of the distribution has an offset of $-2 \mathrm{~km}$ showing that, on average, the method underestimates the peak emission height. In contrast the distribution for method 2 (Fig. 8b) is considerably wider (standard deviation $=4.8 \mathrm{~km}$ ), but has its peak at zero height offset, showing that on average the magnetic field-line method produces the correct answer, but the uncertainty is larger than for method 1 .

The most probable cause of the $2 \mathrm{~km}$ offset in the results from method 1 is that an auroral structure located between the two stations will be projected onto the horizontal plane such that one image will be a mirror image of the other. This can cause the correlation coefficient to be at maximum for a horizontal plane which is not at the height of peak emission. This problem does not occur when projecting onto magnetic field lines as in method 2, which allows the two projections to be aligned. This concept is illustrated in Fig. 1. The left panel shows a cartoon of an auroral arc (black) located between two cameras $\left(s_{1}\right.$ and $\left.s_{2}\right)$. The blue and red curves above the cameras' fields of view indicate the brightness of the arc as observed by $s_{1}$ and $s_{2}$ respectively. The middle panel shows these two brightness curves projected onto a horizontal plane at three different heights, including the peak volume emission height of the auroral arc (middle projection), as for method 1 . The arrows indicate the "up" direction from each projection. It is clear that the shape of each brightness profile is flipped horizontally with respect to the other profile. The right panel of the figure shows the two brightness curves instead projected onto three different magnetic field lines, as in method 2. In this case the two profiles are aligned, and are most highly correlated when the peaks are at the same height, as for the central field line projection in the right panel of Fig. 1.

Figure 9a and $\mathrm{b}$ are histograms showing the distribution of synthetic arc angles for images present in the tails of the distributions in Fig. 8a and b, respectively. Only synthetic images which produce a height value further than 1 standard deviation from the mean are included in these histograms. These show that the vast majority of unreliable results from both methods correspond to analysis of simulated arcs with angles of about $20-55^{\circ}$ (approximately NW-SE aligned). These arcs roughly follow a line connecting the two stations, so within the overlapping region of the images there is a large ambiguity between height and length along the arc (the baseline between Kilpisjärvi and Sodankylä corresponds to an angle of $37.6^{\circ}$ ). When excluding all arcs with angles between $15^{\circ}$ and $75^{\circ}$, both methods become more consistent. Figure 10 shows histograms which are the same as those in Fig. 8, except that only simulated arcs with angles less than $15^{\circ}$ (east-west aligned) or greater than $75^{\circ}$ (north-south aligned) are included. Both distributions are narrower than those for all angles shown in Fig. 8, with standard deviations of $0.7 \mathrm{~km}$ and $2.9 \mathrm{~km}$ for methods 1 and 2, respectively. This shows that both methods are most reliable for arcs which do 

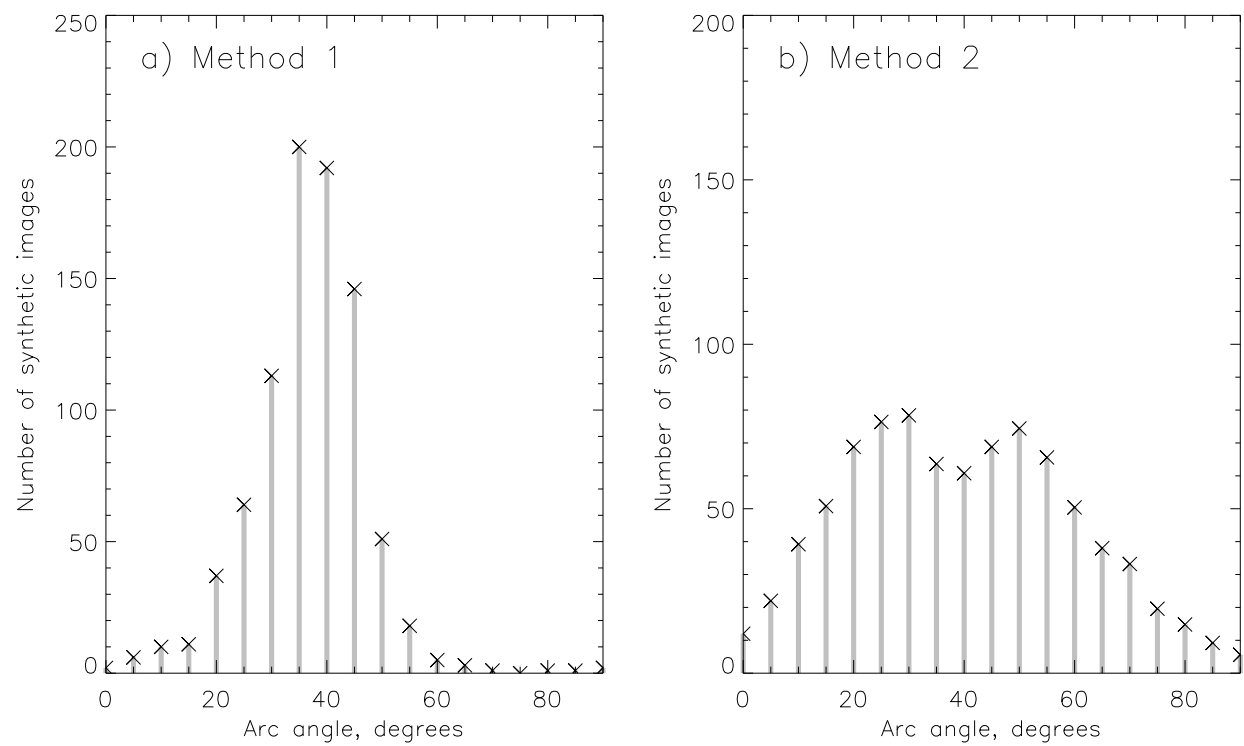

Fig. 9. Histograms showing the distribution of arc angles for images which produce results further than 1 standard deviation from the mean result for (a) method 1 and (b) method 2.
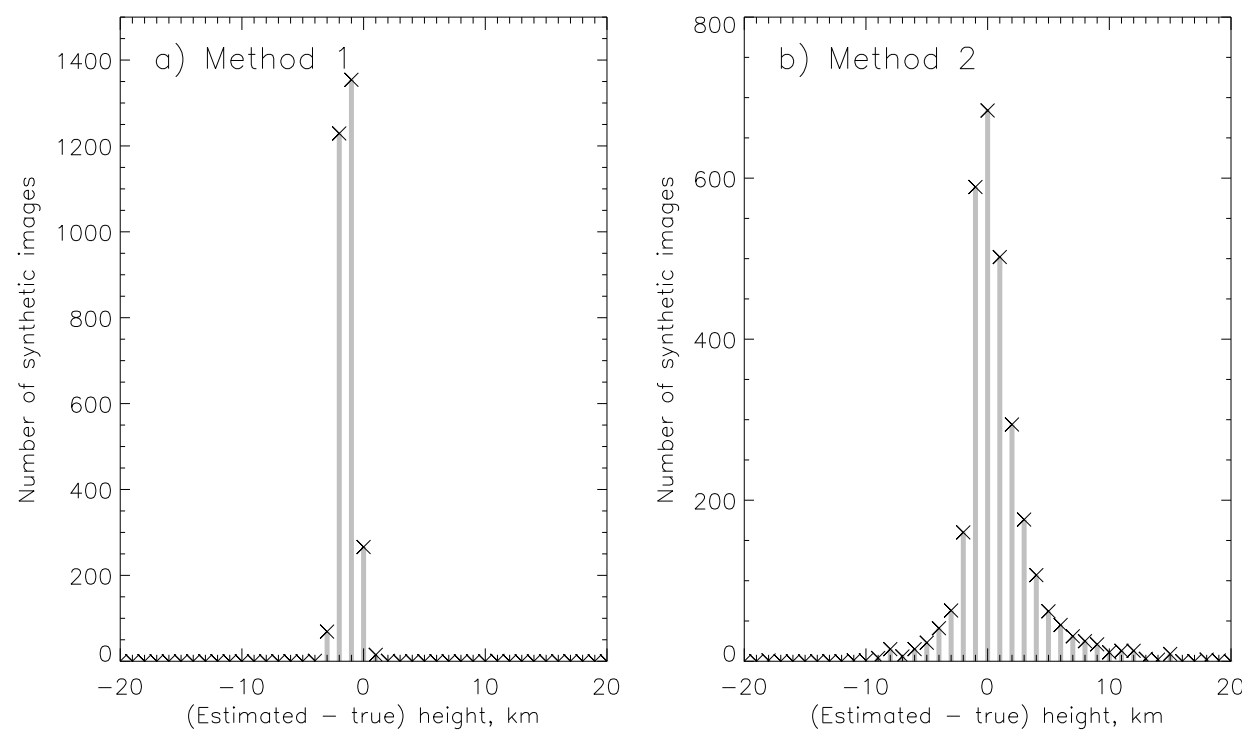

Fig. 10. As Fig. 8 but showing results from only N-S and E-W aligned arcs for (a) method 1 (b) method 2. Standard deviations: (a) 0.7, (b) 2.9 .

not lay on the baseline between the two stations and therefore do not have a large ambiguity between height and length along the arc.

The width of an auroral arc has a substantial effect on the ambiguity between the vertical and horizontal dimensions within the 2-dimensional images of the arc. In this work the two methods have been tested using simulated arcs with widths ranging from $5 \mathrm{~km}$ to $35 \mathrm{~km}$, as described in Sect. 5 . Figure 11 shows how the performance of (a) method 1 and (b) method 2 varies with arc width. Each panel shows seven histograms, one for each arc width tested. The shaded bins are coloured according to the number of image pairs (the scale on the right of the figure). It is clear from panel (a) that method 1 performs consistently regardless of arc width up to the tested limit of $35 \mathrm{~km}$. The histograms show an almost constant bias (estimated height - true height) of about $-2 \mathrm{~km}$. However, panel b) shows that the bias of method 2 does vary with arc width. In most cases the bias is about zero, but the variance and bias of the results increase with increasing arc width, particularly for arcs wider than $25 \mathrm{~km}$. For arcs $35 \mathrm{~km}$ wide the bias of method 2 is $+1.4 \mathrm{~km}$ (an overestimation of the height). For the same arcs the bias of method 1 


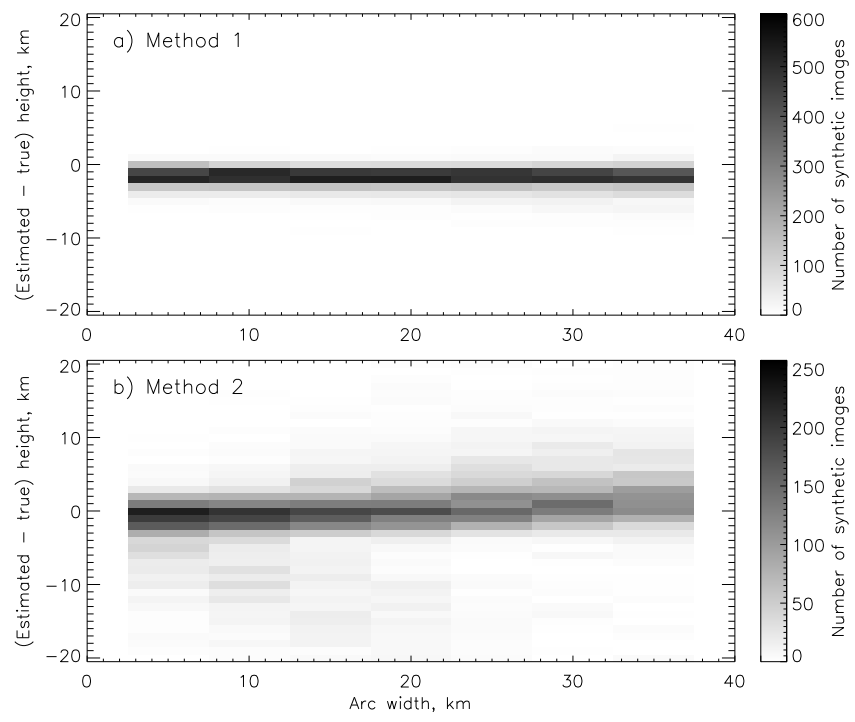

Fig. 11. The effect of the auroral arc width on the performance of (a) method 1 and (b) method 2.

is $-2.4 \mathrm{~km}$. Method 1 implicitly assumes that the aurora is predominantly flat and horizontal, whereas method 2 implicitly assumes that the aurora is made up of rays parallel to the magnetic field. It is therefore logical that method 1 performs well when the aurora is significantly horizontal (such as for $35 \mathrm{~km}$ wide arcs) and method 2 performs well when the aurora is predominantly vertical (such as for thin arcs).

Method 2 involves taking an average profile over multiple field lines, each of which can provide an estimate of the peak emission height. The standard deviation of the height over these field lines is related to the accuracy of the method, and to the actual variation in height across the auroral structure. Figure 12 shows the relationship between this standard deviation and the difference between the estimated and true heights for the set of 9310 synthetic images described above as a 2-dimensional histogram. The shaded bins are coloured according to the scale on the right of the figure. The red dashed line highlights where the standard deviation is equal to the difference between estimated and true height. For the majority of test images, this difference is less than the standard deviation ( $85 \%$ of images, histogram bins below the red dotted line), showing that the standard deviation across "good" test field lines can provide a useful estimate of the uncertainty of the method result. When examining real images, it is important to note that a large standard deviation could result from an auroral structure which does not have one constant peak emission height.

To check that the methods respond to the peak emission height (rather than another property such as mean emission height), synthetic images of the same auroral arc were generated using five different volume emission rate profiles and were analysed using both methods. For each profile the footprints of the auroral rays used to generate the synthetic im-

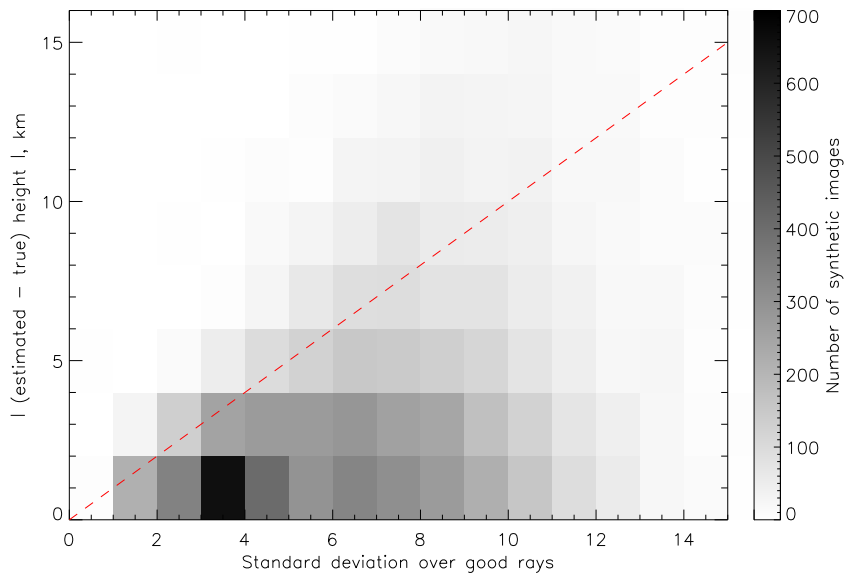

Fig. 12. Two dimensional histogram showing the relationship between the standard deviation of the peak height over all field lines which contribute to the average emission profile in method 2 and the difference between the true peak height and the height estimated by the method.

ages were identical, and formed an east-west aligned arc $350 \mathrm{~km}$ long, $10 \mathrm{~km}$ wide and with a bendiness of 0.1 . All of the profiles have a peak emission height of $110 \mathrm{~km}$, but each has a different mean emission height. The five profiles are shown in Fig. 13. The results from the two methods are shown on the right of the figure as diamonds, colour-coded to match the height profiles to which they correspond. The same results are shown in the magnified panel (far right) for clarity, where the standard deviations over "good" test field lines used in method 2 (as discussed above) are plotted as error bars with a total length of twice the standard deviation. Both methods respond quite well to the peak emission height for all profiles, but method 2 is clearly less susceptible to variations in the shape of the profile and produces the best results. For all profiles the peak emission height is within the error estimate for method 2. The dark blue-coloured profile was used in the generation of the 9310 synthetic image pairs described in Sect. 5. For this profile method 1 underestimates the peak emission height, which is consistent with the results discussed earlier, but for most other profiles it produces an overestimate.

While this study concentrates on analysing images of an arc centred at the midpoint between the Kilpisjärvi and Sodankylä stations, a short series of tests on arcs located to the north or south of this point have also been performed. The results are shown as a set of histograms in Fig. 14 for method 1 (panel a) and method 2 (panel b), with shaded bins coloured according to the number of image pairs (scales on the right). Seven points were tested between $67.47^{\circ} \mathrm{N}$ and $68.97^{\circ} \mathrm{N}$, all at the same longitude of $23.59^{\circ} \mathrm{E}$. The locations of the Sodankylä (SOD) and Kilpisjärvi (KIL) stations are marked with dashed lines. For each latitude tested a set of 1000 random synthetic image pairs was analysed using both methods, with arc properties (length, width, bendiness) in the 


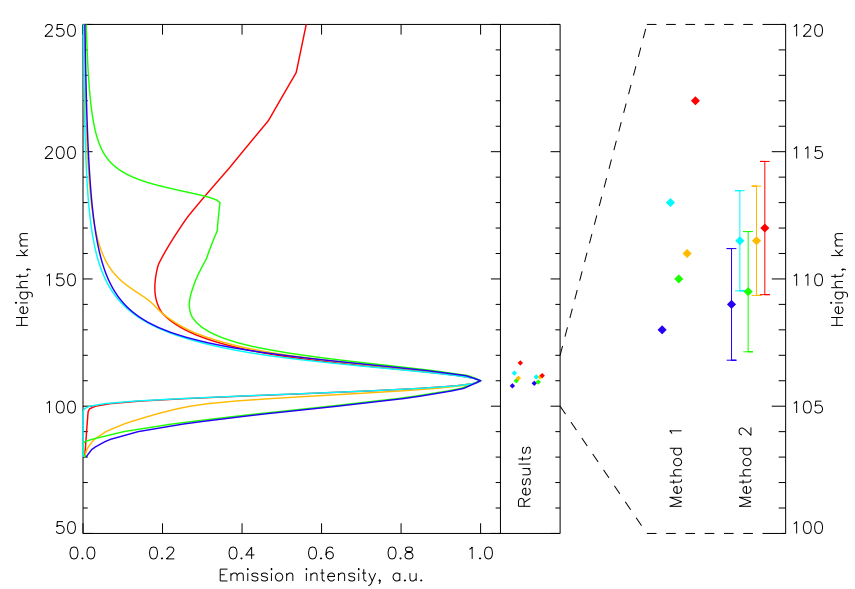

Fig. 13. Five different volume emission rate profiles used to test whether the methods respond to the peak emission height. The results from both methods are shown on the right of this figure.

same ranges as described in Sect. 5. All of the synthetic arcs were approximately east-west aligned. The results of these tests show that method 1 performs consistently for all arc locations which have been tested. However, while method 2 is successful for arcs located approximately halfway between the two stations, it does not perform well for arcs located a few tenths of a degree to the south of either station. In these cases the arc is in the magnetic zenith as viewed from one of the stations, and therefore many auroral rays appear as dots or very short lines in the corresponding image. It is not possible to extract accurate emission profiles from test field lines close to the magnetic zenith, and so method 2 fails to provide a result or performs poorly in these cases. The results of method 2 appear to be biased for arcs located directly above the Kilpisjärvi station, but the reason for this is unclear. It is possible that the arcs are so close to the horizon of the Sodankylä images that the pixel resolution is too low to produce reliable altitude profiles from test field lines. It is not possible to analyse arcs located far to the north or south of both stations, as the separation of the stations is too large. The tests performed here are not exhaustive, and further analysis is required to fully assess the performance of the methods for extreme arc locations. However, in most cases images suitable for analysis will contain arcs located between the stations.

In addition to analysis of the set of synthetic images, the methods have been trialed on a short set of real images of $557.7 \mathrm{~nm}$ emission acquired by the Abisko and Kilpisjärvi cameras. Abisko is approximately $110 \mathrm{~km}$ south-west of Kilpisjärvi. Examples from this image set, which displays a single quiet arc, are shown in Fig. 15. The top panel shows an image from Abisko, and the bottom panel shows the coincident image from Kilpisjärvi. Although this is only a single short event it is suitable as a simple initial test for the methods presented in this paper as the arc is stable, approximately east-west aligned, and there are no other auroral structures in

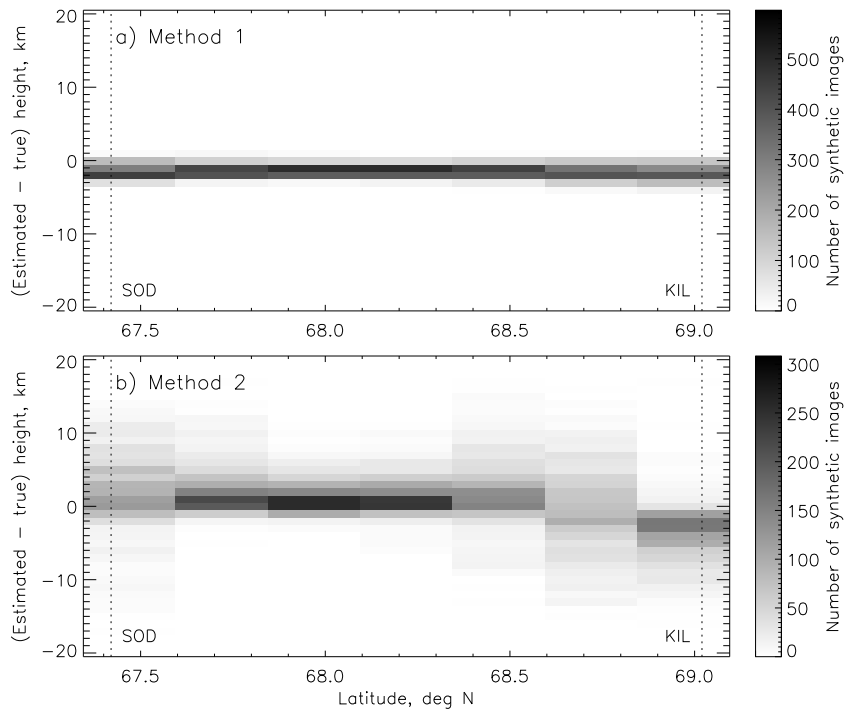

Fig. 14. Results from the analysis of synthetic arcs located north or south of the midpoint between the Sodankylä and Kilpisjärvi stations. The same images were analysed using both methods, and results are shown as histograms for (a) method 1 and (b) method 2.

the images which could confuse the analysis. The variation in height along the length of the arc present in this image set was analysed by Sangalli et al. (2011) using a triangulation method. While our automatic methods give only a single value for the height of the arc, it is useful to compare the results to those from the more manual method employed by Sangalli et al. (2011). The results obtained from this analysis are shown in Fig. 16. Methods 1 and 2 are shown as black open diamonds and green solid diamonds, respectively. The green bars show the standard deviation of the peak height over all field lines which contribute to the average profile in method 2 (total bar length is equal to twice the standard deviation). The results of Sangalli et al. (2011) are shown as red bars to indicate the range of heights identified across the arc. As with the analysis of the synthetic images, it is clear that method 1 produces lower height values than method 2 . The results from method 2 consistently agree more closely with the more comprehensive method employed by Sangalli et al. (2011). The small uncertainty estimates associated with the method 2 results suggest that the method works well for real data as well as for synthetic images; the uncertainty estimates in Fig. 16 are slightly higher than for the synthetic images, but this is to be expected as the height along the arc is known to vary, whereas the simulated arcs have a constant peak height. It should be noted that some of the images at the start and end of the event produce results using method 1 but not using method 2 (not shown in Fig. 16). At these times the arc was weak. When analysing these images using method 2, either no field lines led to high correlation between the two images or none of the emission profiles on the magnetic field lines were found to have a clear peak, and 

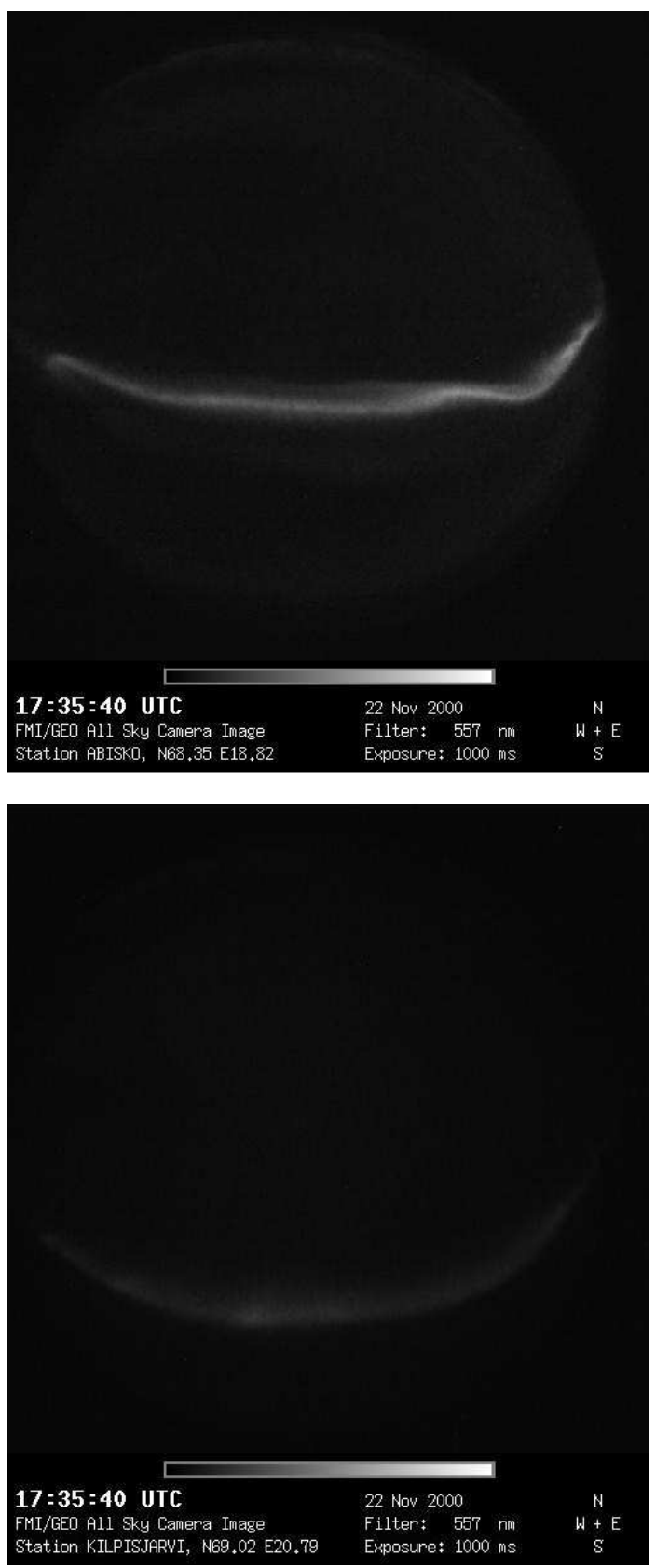

Fig. 15. Images acquired simultaneously by all-sky cameras in Abisko (top) and Kilpisjärvi (bottom). Both images show the same auroral arc.

therefore no result was obtained. At these times the results using method 1 are unreliable. In order to avoid such unreliable results, a test could be applied to images before analysis to ensure they contain aurora with a sufficient brightness. Available machine vision methods would be able to perform this test with an accuracy greater than $90 \%$, although this is not a trivial process (Syrjäsuo and Partamies, 2011).

The clustering process used in method 2 to select a region of the image for analysis could bias the results, as it restricts

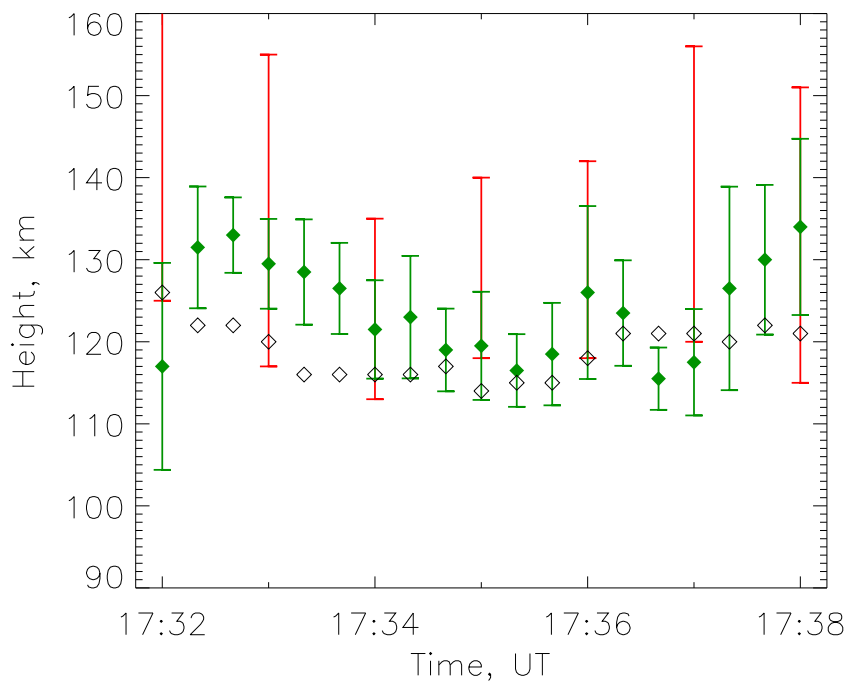

Fig. 16. The peak emission height of an auroral arc on 22 November 2000 found using method 1 (black), method 2 (green) and by the manual method of Sangalli et al. (2011) (red). In all cases the Abisko and Kilpisjärvi stations were used.

the analysis to a bright structure within the image. It may be expected that the peak emission height of the aurora is related to its brightness (although this is not yet clear). However, this bias will only become apparent for images with aurora covering a very large portion of the image, for example diffuse aurora or a system of many parallel arcs. Under these conditions it is likely that the method would fail anyway, as the ambiguity between the horizontal and vertical dimensions inherent to a 2-dimensional image would be large. It is also unlikely that method 1 would produce a strong correlation between images in these cases.

\section{Conclusions}

This paper presents a new method for finding the peak emission height of the aurora using simultaneous all-sky camera images from a pair of stations. This method has been developed and tested to evaluate its suitability for a statistical study into the height of the aurora using hundreds of thousands of all-sky camera images from northern Finland. For a statistical study of this magnitude, it is vitally important to have a fast and reliable analysis method which is fully automatic. Another method (horizontal plane) has also been tested in the same way as the new method.

The test results reported here show that of the two methods, the horizontal plane method is less affected by the orientation and location of an auroral structure. However, the field line method measures the peak emission height with little dependence on the shape of the volume emission rate profile, whereas the horizontal plane method is biased by emission above and below the peak height. Neither of the two 
methods are perfect, but both are a marked improvement on earlier methods when considering speed, accuracy and level of automation.

For typical arcs located between the two stations, the field line method (method 2) is recommended, as it responds well to the peak emission height and produces the correct answer on average. It also provides a useful uncertainty estimate. For arcs which are located close to the horizon or in the magnetic zenith of one of the images, the horizontal plane method (method 1 ) is recommended. This method is also recommended for very wide arcs or aurora which could be considered as predominantly flat (parallel to the Earth's surface) rather than parallel to the magnetic field. The new field line method is most apropriate for a statistical study of quiet arcs over Lapland, but either method would provide valuable new information on the peak height under different conditions for a large enough data set.

Future work will investigate the possibilty of utilising simultaneous observations from several (more than two) MIRACLE stations to ensure accurate results from arcs with any orientation or location. It may also be possible to apply a combination of the two methods to obtain greater accuracy than either method alone could provide. A hybrid method could also give information about the shape of the volume emission rate profile, as the results of method 1 and method 2 are influenced by the profile shape in different ways. This would require extensive testing. The synthetic images used here contain only a single arc located between the two stations with a constant peak emission height, whereas in reality all-sky camera images often contain multiple auroral structures. Therefore, the methods should be tested on more complex synthetic images and synthetic arcs with varying peak emission height. More tests should also be performed on real images for which the peak emission height can be determined using other more precise methods or instruments.

Acknowledgements. This work was financially supported by the Academy of Finland (grant number 128553). The MIRACLE network is operated as an international collaboration under the leadership of the Finnish Meteorological Institute. The authors thank the participants of the 38th Annual European Meeting on Atmospheric Studies by Optical Methods (38am) for useful comments and suggestions.

Edited by: A. Benedetto

\section{References}

Ashrafi, M., Kosch, M. J., and Kaila, K.: Height triangulation of artificial optical emissions in the F-layer, in: Proceedings of the 31st Annual European Meeting on Atmospheric Studies by Optical Methods, and 1st International Riometer Workshop, 2228 August 2004, Ambleside, UK, 8-16, http://spears.lancs.ac.uk/ publications/31am_proceedings.pdf, 2005.
Aso, T., Ejiri, M., Urashima, A., Miyaoka, H., Steen, Å., Brändström, U., and Gustavsson, B.: First results of auroral tomography from ALIS-Japan multi-station observations in March, 1995, Earth, Planet. Space, 50, 81-86, 1998.

Boyd, J. S., Belon, A. E., and Romick, G. J.: Latitude and Time Variations in Precipitated Electron Energy Inferred from Measurements of Auroral Heights, J. Geophys. Res., 76, 7694-7700, 1971.

Brandy, J. H. and Hill, J. E.: Rapid Determination of Auroral Heights, Can. J. Phys., 42, 1813-1819, doi:10.1139/p64-165, 1964.

Dahlgren, H., Ivchenko, N., Lanchester, B., Ashrafi, M., Whiter, D., Marklund, G., and Sullivan, J.: First direct optical observations of plasma flows using afterglow of $\mathrm{O}^{+}$in discrete aurora, J. Atmos. Sol. Terr. Phys., 71, 228-238, doi:10.1016/j.jastp.2008.11.015, 2009.

Deehr, C. S., Rees, M. H., Belon, A. E. H., Romick, G. J., and Lummerzheim, D.: Influence of the ionosphere on the altitude of discrete auroral arcs, Ann. Geophys., 23, 759-766, doi:10.5194/angeo-23-759-2005, 2005.

Frey, S., Frey, H. U., Carr, D. J., Bauer, O. H., and Haerendel, G.: Auroral emission profiles extracted from three-dimensionally reconstructed arcs, J. Geophys. Res., 101, 21731-21741, 1996.

Gustavsson, B.: Three Dimensional Imaging of Aurora and Airglow, PhD, Swedish Institute of Space Physics (IRF), Kiruna, Sweden, http://www.irf.se/ bjorn/thesis/thesis.html, (IRF Sci. Report 267), ISBN: 91-7191-878-7, 2000.

Harang, L.: The Aurorae, Chapman \& Hall, London, 1951.

Hedin, A. E.: Extension of the MSIS thermosphere model into the middle and lower atmosphere, J. Geophys. Res., 96, 1159-1172, doi:10.1029/90JA02125, 1991.

Jackel, B. J., Creutzberg, F., Donovan, E. F., and Cogger, L. L.: Triangulation of Auroral Red-Line Emission Heights, in: Proceedings of the 28th Annual European Meeting on Atmospheric Studies by Optical Methods, 19-24 August 2001, Oulu, Finland, edited by: Kaila, K. U., Jussila, J. R. T., and Holma, H., no. 92 in Sodankylä Geophysical Observatory Publications, 97100, 2003.

Judge, R. J. R.: Electron Excitation and Auroral Emission Parameters, Planet. Space Sci., 20, 2081-2092, 1972.

Kaila, K. U.: Determination of the energy of auroral electrons by the measurements of the emission ratio and altitude of aurorae, Planet. Space Sci., 37, 341-349, 1989.

Knudsen, D. J., Donovan, E. F., Cogger, L. L., Jackel, B., and Shaw, W. D.: Width and structure of mesoscale optical auroral arcs, Geophys. Res. Lett., 28, 705-708, 2001.

Maus, S., Macmillan, S., Chernova, T., Choi, S., Dater, D., Golovkov, V., Lesur, V., Lowes, F., Lühr, H., Mai, W., McLean, S., Olsen, N., Rother, M., Sabaka, T., Thomson, A., and Zvereva, T.: The 10th-Generation International Geomagnetic Reference Field, Geophys. J. Int., 161, 561-565, doi:10.1111/j.1365246X.2005.02641.x, 2005.

McEwen, D. J. and Montalbetti, R.: Parallactic Measurements on Aurorae over Churchill, Canada, Can. J. Phys., 36, 1593-1600, doi:10.1139/p58-161, 1958.

Obuchi, Y., Sakanoi, T., Asamura, K., Yamazaki, A., Kasaba, Y., Hirahara, M., Ebihara, Y., and Okano, S.: Fine-scale dynamics of black auroras obtained from simultaneous imaging and particle observations with the Reimei satellite, J. Geophys. Res., 116, 
A00K07, doi:10.1029/2010JA016321, 2011.

Partamies, N., Syrjäsuo, M., Donovan, E., Connors, M., Charrois, D., Knudsen, D., and Kryzanowsky, Z.: Observations of the auroral width spectrum at kilometre-scale size, Ann. Geophys., 28, 711-718, doi:10.5194/angeo-28-711-2010, 2010.

Rees, M. H.: A Method for Determining the Height and Geographical Position of an Auroral Arc from One Observing Station, J. Geophys. Res., 68, 175-183, 1963a.

Rees, M. H.: Auroral Ionization and Excitation by Incident Energetic Electrons, Planet. Space Sci., 11, 1209-1218, 1963b.

Roach, F. E., Moore, J. G., Bruner Jr., E. C., Cronin, H., and Silverman, S. M.: The Height of Maximum Luminosity in an Auroral Arc, J. Geophys. Res., 65, 3575-3580, 1960.

Romick, G. J. and Belon, A. E.: The Spatial Variation of Auroral Luminosity-I. The Behavior of Synthetic Model Auroras, Planet. Space Sci., 15, 475-493, 1967a.

Romick, G. J. and Belon, A. E.: The Spatial Variation of Auroral Luminosity-II. Determination of Volume Emission Rate Profiles, Planet. Space Sci., 15, 1695-1716, $1967 \mathrm{~b}$.

Sandholt, P.-E., Egeland, A., Henriksen, K., Smith, R., and Sweeney, P.: Optical Measurements of a Nightside Poleward Expanding Aurora, J. Atmos. Terr. Phys., 44, 71-79, 1982.

Sangalli, L., Gustavsson, B., Partamies, N., and Kauristie, K.: Estimating the Peak Auroral Emission Altitude from All-sky Images, Óptica Pura y Aplicada, 44, 593-598, 2011.

Semeter, J., Zettergren, M., Diaz, M., and Mende, S.: Wave dispersion and the discrete aurora: New constraints derived from high-speed imagery, J. Geophys. Res., 113, A12208, doi:10.1029/2008JA013122, 2008.
Sigernes, F., Moen, J., Lorentzen, D. A., Deehr, C. S., Smith, R., Øieroset, M., Lybekk, B., and Holtet, J.: SCIFER-Height measurements of the midmorning aurora, Geophys. Res. Lett., 23, 1889-1892, 1996.

Störmer, C.: The Polar Aurora, The Clarendon Press, Oxford, 1955. Syrjäsuo, M. T. and Donovan, E. F.: Using Relevance Feedback in Retrieving Auroral Images, in: Proceedings of the Fourth IASTED International Conference on Computational Intelligence, Calgary, Canada, 4-6 July, edited by: Hamza, M. H., 420 425, IASTED/ACTA Press, 2005.

Syrjäsuo, M. and Partamies, N.: Numeric Image Features for Detection of Aurora, Geosci. Remote Sens. Lett., IEEE, 9, 176-179, doi:10.1109/LGRS.2011.2163616, 2011.

Syrjäsuo, M., Pulkkinen, T. I., Janhunen, P., Viljanen, A., Pellinen, R. J., Kauristie, K., Opgenoorth, H. J., Wallman, S., Eglitis, P., Karlsson, P., Amm, O., Nielsen, E., and Thomas, C.: Observations of Substorm Electrodynamics Using the MIRACLE Network, in: Proceedings of the International Conference on Substorms-4, Lake Hamana, Japan, 9-13 March, edited by: Kokubun, S. and Kamide, Y., 111-114, Terra Scientific Publishing, Tokyo, Japan, 1998.

Tanaka, Y.-M., Aso, T., Gustavsson, B., Tanabe, K., Ogawa, Y., Kadokura, A., Miyaoka, H., Sergienko, T., Brändström, U., and Sandahl, I.: Feasibility study on Generalized-Aurora Computed Tomography, Ann. Geophys., 29, 551-562, doi:10.5194/angeo29-551-2011, 2011.

Whiter, D. K., Lanchester, B. S., Gustavsson, B., Ivchenko, N., Sullivan, J. M., and Dahlgren, H.: Small-scale structures in flickering aurora, Geophys. Res. Lett., 35, L23103, doi:10.1029/2008GL036134, 2008. 